\title{
Theory of the Dielectric Susceptibility of Liquid Crystals with Bent-Core Molecules
}

\author{
A. KAPANOWSKI* \\ Institute of Physics, Jagiellonian University, W.S. Reymonta 4, 30-059 Cracow, Poland
}

(Received November 24, 2010; in final form April 19, 2011)

\begin{abstract}
Statistical theory of the dielectric susceptibility of polar liquid crystals is proposed. The molecules are calamitic or bent-core but the permanent dipole moment is perpendicular to the molecule long axis. The ordering of the phase is described by means of the mean-field theory based on the Maier-Saupe approach. The theory is used to calculate the temperature dependence of the order parameters and the susceptibilities. The phase diagram with four phases is obtained: isotropic, uniaxial nematic, uniaxial ferroelectric, and biaxial ferroelectric. Four critical points are predicted.
\end{abstract}

PACS: $61.30 . \mathrm{Cz}, 77.84 . \mathrm{Nh}$

\section{Introduction}

Liquid crystals are always built from anisotropic molecules [1]. For a long time calamitic or discotic molecules were considered as appropriate molecules for the formation of liquid crystal phases. But it appeared that bent-core molecules can lead to many interesting phenomena related to polarity and chirality [2]. Phases and phase transitions that can take place in the bent-core systems were studied by Lubensky and Radzihovsky [3]. They showed that to completely characterize phases a third-rank tensor order parameter is necessary in addition to the vector and the nematic (second-rank) tensor order parameters. Twelve different liquid phases were identified and many symmetry-allowed transitions among them were analyzed in detail. The bent-core molecules were studied also by means of computer simulations [4-7].

We would like to study a simple model that can capture main features of bent-core molecules. Let us consider a system of $N$ molecules contained in a volume $V$ at temperature $T$. We assume that the potential energy of the interactions $V\left(R_{1}, R_{2}\right)$ depends only on the molecule orientations $R_{1}$ and $R_{2}$. The orientation of a molecule is described by the three Euler angles $R=(\phi, \theta, \psi)$ or by the three orthonormal vectors $(\boldsymbol{l}, \boldsymbol{m}, \boldsymbol{n})$. The potential energy of interactions has the form

$$
V\left(R_{1}, R_{2}\right)=v_{0}+v_{1} P_{1}\left(\boldsymbol{l}_{1} \cdot \boldsymbol{l}_{2}\right)+v_{2} P_{2}\left(\boldsymbol{n}_{1} \cdot \boldsymbol{n}_{2}\right),
$$

where $P_{j}$ are the Legendre polynomials, the vector $\boldsymbol{n}$ determines the long molecule axis. If $v_{1}=0$ then the Maier-Saupe theory is recovered, where the nematic phase is present for $v_{2}<0$. The $v_{1}$ term breaks $D_{\infty h}$ symmetry and it partly describes bent-core molecules with $C_{2 v}$ symmetry. On the other hand, the $v_{1}$ term can be connected with the permanent electric dipole $\boldsymbol{p}=p \boldsymbol{l}$

* e-mail: andrzej.kapanowski@uj.edu.pl of a molecule because many bent-core molecules have a transverse dipole which bisects the bend angle [7].

Our aim is to create the theory of the susceptibility which is a tensor in the case of anisotropic phases

$$
\epsilon_{0} \chi_{\alpha \beta}=\frac{\partial P_{\alpha}}{\partial E_{\beta}},
$$

where $P$ and $E$ are the polarization and the electric field, respectively. In the first approximation, the polarization can be written as

$$
P_{\alpha}=\frac{N p}{V}\left\langle\bar{l}_{\alpha}\right\rangle,
$$

where $p\left\langle\bar{l}_{\alpha}\right\rangle$ is the average value of the dipole component in the $\alpha$-direction in the presence of the electric field. Two different averages are involved here. The bar refers to the potential energy of the dipole moment in the electric field and the brackets to the nematic potential. The potential energy of the dipole $\boldsymbol{p}$ in the electric field $\boldsymbol{E}$ is $\boldsymbol{p} \cdot \boldsymbol{E}$. The linear approximation is applied to resolve the influence of the electric field

$$
\left\langle\bar{l}_{\alpha}\right\rangle=\left\langle l_{\alpha}\left(1+\beta p l_{\beta} E_{\beta}\right)\right\rangle=\beta p\left\langle l_{\alpha} l_{\beta}\right\rangle E_{\beta},
$$

where $1 / \beta=k_{\mathrm{B}} T$. Thus the susceptibility has the form

$$
\epsilon_{0} \chi_{\alpha \beta}=\frac{N p^{2} \beta}{V}\left\langle l_{\alpha} l_{\beta}\right\rangle .
$$

The components of the susceptibility in the nematic phase oriented along the $z$ axis are

$$
\begin{aligned}
& \epsilon_{0} \chi_{x x}=\epsilon_{0} \chi_{y y}=\frac{N p^{2} \beta(2+S)}{6 V}, \\
& \epsilon_{0} \chi_{z z}=\frac{N p^{2} \beta(1-S)}{3 V},
\end{aligned}
$$

where $S=\left\langle P_{2}\left(n_{z}\right)\right\rangle$ is the order parameter. More advanced calculations were given by Maier and Meier [8] who extended the Onsager theory of the susceptibility to nematic liquid crystals. If we neglect the induced polar- 
ization we can write their results as

$$
\begin{aligned}
& \epsilon_{0} \chi_{x x}=\epsilon_{0} \chi_{y y}=\left(\frac{3 \tilde{\chi}+3}{2 \tilde{\chi}+3}\right) \frac{N p^{2} \beta(2+S)}{6 V}, \\
& \epsilon_{0} \chi_{z z}=\frac{3 \tilde{\chi}+3}{2 \tilde{\chi}+3} \frac{N p^{2} \beta(1-S)}{3 V},
\end{aligned}
$$

where $\tilde{\chi}$ is the average susceptibility of the phase.

The organization of this paper is as follows: In Sect. 2 the mean-field theory of the phase ordering is provided. In Sect. 3 the formulae for the susceptibility are derived in the case of the nematic and the ferroelectric phase. Section 4 is devoted to some applications of the presented theory. Section 5 contains a summary. Appendix A provides the definitions and main properties of the basic functions.

\section{Mean-field theory}

The potential energy of molecular interactions (1) can be rewritten in the form

$$
V\left(R_{1}, R_{2}\right)=v_{0}+v_{1} E_{11}^{(1)}\left(R_{2}^{-1} R_{1}\right)+v_{2} E_{00}^{(2)}\left(R_{2}^{-1} R_{1}\right),
$$

where $E_{\mu \nu}^{(j)}$ are the basic functions defined in Appendix A [9]. The basic functions will facilitate many future calculations. The molecular orientation in the phase is described by the distribution function

$$
\int \mathrm{d} R f(R)=1 \text {. }
$$

The mean of any function $A=A(R)$ can be calculated as

$$
\langle A\rangle \equiv \int \mathrm{d} R f(R) A(R) .
$$

The state of the system is described by a series of order parameters $\left\langle E_{\mu \nu}^{(j)}\right\rangle$ but the most important have $j=1$ or $j=2$. The internal energy of the system is

$$
U=\frac{N}{2} \int \mathrm{d} R_{1} \mathrm{~d} R_{2} f\left(R_{1}\right) f\left(R_{2}\right) V\left(R_{1}, R_{2}\right),
$$

whereas the entropy of the system has the form

$$
S=-k_{\mathrm{B}} N \int \mathrm{d} R f(R) \ln \left(f(R) C_{N}\right) .
$$

The energy of permanent dipole moments in the electric field $\boldsymbol{E}$ is

$$
U_{E}=N\langle\boldsymbol{p} \cdot \boldsymbol{E}\rangle .
$$

The total free energy of the system is the sum

$$
F_{\text {tot }}=U+U_{E}-T S .
$$

In the mean-field approximation a potential energy is

$$
W(R)=\sum_{j} \sum_{\mu \nu} w_{\mu \nu}^{(j)} E_{\mu \nu}^{(j)}(R),
$$

where the unknown coefficients $w_{\mu \nu}^{(j)}$ depend on the phase ordering and temperature. The distribution function can be found from the Boltzmann distribution

$$
f(R)=\exp (-\beta W(R)) / Z,
$$

where $Z$ is a normalization constant. The consistency condition

$$
W\left(R_{1}\right)=\int \mathrm{d} R_{2} f\left(R_{2}\right) V\left(R_{1}, R_{2}\right)-\boldsymbol{p}\left(R_{1}\right) \cdot \boldsymbol{E}
$$

leads to equations

$$
\begin{aligned}
& w_{\mu \nu}^{(1)}=v_{1}\left\langle E_{\mu 1}^{(1)}\right\rangle \delta_{\nu 1}-p \delta_{\nu 1}\left(E_{x} \delta_{1 \mu}-E_{y} \delta_{-1 \mu}+E_{z} \delta_{0 \mu}\right), \\
& w_{\mu \nu}^{(2)}=v_{2}\left\langle E_{\mu 0}^{(2)}\right\rangle \delta_{\nu 0} .
\end{aligned}
$$

It is useful to introduce the dimensionless parameters $S_{\mu \nu}^{(j)}=-\beta w_{\mu \nu}^{(j)}$ for $j>0$

$$
\ln f(R)=\sum_{j} \sum_{\mu \nu} S_{\mu \nu}^{(j)} E_{\mu \nu}^{(j)}(R) .
$$

$S_{00}^{(0)}$ is responsible for the normalization and it depends on other $S_{\mu \nu}^{(j)}$ with $j>0$ :

$$
\begin{aligned}
& S_{00}^{(0)}=-\ln \left(\int \mathrm{d} R \exp \left(\sum_{j>0} \sum_{\mu \nu} S_{\mu \nu}^{(j)} E_{\mu \nu}^{(j)}(R)\right)\right), \\
& U_{\mu \nu}^{11} \equiv\left\langle E_{\mu 1}^{(1)} E_{\nu 1}^{(1)}\right\rangle-\left\langle E_{\mu 1}^{(1)}\right\rangle\left\langle E_{\nu 1}^{(1)}\right\rangle, \\
& U_{\mu \nu}^{12} \equiv\left\langle E_{\mu 1}^{(1)} E_{\nu 0}^{(2)}\right\rangle-\left\langle E_{\mu 1}^{(1)}\right\rangle\left\langle E_{\nu 0}^{(2)}\right\rangle, \\
& U_{\mu \nu}^{22} \equiv\left\langle E_{\mu 0}^{(2)} E_{\nu 0}^{(2)}\right\rangle-\left\langle E_{\mu 0}^{(2)}\right\rangle\left\langle E_{\nu 0}^{(2)}\right\rangle, \\
& \frac{\partial S_{00}^{(0)}}{\partial S_{\mu \nu}^{(j)}}=-\left\langle E_{\mu \nu}^{(j)}\right\rangle, \quad U_{\mu \nu}^{j k}=U_{\nu \mu}^{k j} .
\end{aligned}
$$

Now Eqs. (20), (21) have the form

$$
\begin{aligned}
& S_{\mu \nu}^{(1)}+\beta v_{1}\left\langle E_{\mu 1}^{(1)}\right\rangle \delta_{\nu 1} \\
& \quad=\beta p \delta_{\nu 1}\left(E_{x} \delta_{1 \mu}-E_{y} \delta_{-1 \mu}+E_{z} \delta_{0 \mu}\right), \\
& S_{\mu \nu}^{(2)}+\beta v_{2}\left\langle E_{\mu 0}^{(2)}\right\rangle \delta_{\nu 0}=0 .
\end{aligned}
$$

The solution is orientationally stable only if the matrix

$$
\left[U_{\mu \nu}^{j k}+\sum_{l>0} \sum_{\rho} \beta v_{l} U_{\mu \rho}^{j l} U_{\nu \rho}^{k l}\right]
$$

is positive definite. The isotropic phase is orientationally stable if $\beta v_{1}>-3$ and $\beta v_{2}>-5$.

\section{Dielectric susceptibility}

The dielectric susceptibility tensor is defined by Eq. (2) and we calculate the orientational polarization as

$$
P_{\alpha}=\left\langle l_{\alpha}\right\rangle N p / V .
$$

Let us note that the polarization depends on the electric field via the distribution function. The components of the susceptibility are

$$
\epsilon_{0} \chi_{x x}=\frac{N p}{V}\left[\sum_{\mu} U_{1 \mu}^{11} \frac{\partial S_{\mu 1}^{(1)}}{\partial E_{x}}+\sum_{\mu} U_{1 \mu}^{12} \frac{\partial S_{\mu 0}^{(2)}}{\partial E_{x}}\right],
$$




$$
\begin{aligned}
& \epsilon_{0} \chi_{y y}=\frac{N p}{V}(-1) \\
& \times\left[\sum_{\mu} U_{-1 \mu}^{11} \frac{\partial S_{\mu 1}^{(1)}}{\partial E_{y}}+\sum_{\mu} U_{-1 \mu}^{12} \frac{\partial S_{\mu 0}^{(2)}}{\partial E_{y}}\right], \\
& \epsilon_{0} \chi_{z z}=\frac{N p}{V}\left[\sum_{\mu} U_{0 \mu}^{11} \frac{\partial S_{\mu 1}^{(1)}}{\partial E_{z}}+\sum_{\mu} U_{0 \mu}^{12} \frac{\partial S_{\mu 0}^{(2)}}{\partial E_{z}}\right],
\end{aligned}
$$

where the derivatives are calculated from the equations

$$
\begin{aligned}
& \frac{\partial S_{\mu 1}^{(1)}}{\partial E_{x}}+\beta v_{1}\left[\sum_{\rho} U_{\mu \rho}^{11} \frac{\partial S_{\rho 1}^{(1)}}{\partial E_{x}}+\sum_{\rho} U_{\mu \rho}^{12} \frac{\partial S_{\rho 0}^{(2)}}{\partial E_{x}}\right] \\
& \quad=\beta p \delta_{1 \mu}, \\
& \frac{\partial S_{\mu 0}^{(2)}}{\partial E_{x}}+\beta v_{2}\left[\sum_{\rho} U_{\mu \rho}^{21} \frac{\partial S_{\rho 1}^{(1)}}{\partial E_{x}}+\sum_{\rho} U_{\mu \rho}^{22} \frac{\partial S_{\rho 0}^{(2)}}{\partial E_{x}}\right] \\
& \quad=0, \\
& \frac{\partial S_{\mu 1}^{(1)}}{\partial E_{y}}+\beta v_{1}\left[\sum_{\rho} U_{\mu \rho}^{11} \frac{\partial S_{\rho 1}^{(1)}}{\partial E_{y}}+\sum_{\rho} U_{\mu \rho}^{12} \frac{\partial S_{\rho 0}^{(2)}}{\partial E_{y}}\right] \\
& \quad=-\beta p \delta_{-1 \mu}, \\
& \frac{\partial S_{\mu 0}^{(2)}}{\partial E_{y}}+\beta v_{2}\left[\sum_{\rho} U_{\mu \rho}^{21} \frac{\partial S_{\rho 1}^{(1)}}{\partial E_{y}}+\sum_{\rho} U_{\mu \rho}^{22} \frac{\partial S_{\rho 0}^{(2)}}{\partial E_{y}}\right] \\
& \quad=0, \\
& \frac{\partial S_{\mu 1}^{(1)}}{\partial E_{z}}+\beta v_{1}\left[\sum_{\rho} U_{\mu \rho}^{11} \frac{\partial S_{\rho 1}^{(1)}}{\partial E_{z}}+\sum_{\rho} U_{\mu \rho}^{12} \frac{\partial S_{\rho 0}^{(2)}}{\partial E_{z}}\right] \\
& \quad=\beta p \delta_{0 \mu}, \\
& \frac{\partial S_{\mu 0}^{(2)}}{\partial E_{z}}+\beta v_{2}\left[\sum_{\rho} U_{\mu \rho}^{21} \frac{\partial S_{\rho 1}^{(1)}}{\partial E_{z}}+\sum_{\rho} U_{\mu \rho}^{22} \frac{\partial S_{\rho 0}^{(2)}}{\partial E_{z}}\right] \\
& \quad=0 .
\end{aligned}
$$

Now we are in the position to discuss the results for possible phases.

\subsection{The isotropic phase}

For the zero field all order parameters are equal to zero. For the nonzero field the phase has the symmetry $C_{\infty v}$ (the symmetry of the electric field):

$$
\epsilon_{0} \chi_{x x}=\epsilon_{0} \chi_{y y}=\epsilon_{0} \chi_{z z}=\frac{N p^{2} \beta}{V\left(3+\beta v_{1}\right)} .
$$

For $v_{1}<0$ we get the Curie-Weiss law describing the divergence of $\chi$ when we approach the Curie temperature from above. For $v_{1}>0$ the susceptibility is finite.

\subsection{The uniaxial nematic phase}

For the zero field the phase has the symmetry $D_{\infty h}$ and the parameter $S_{00}^{(2)}$ is nonzero for the phase oriented along the $z$ axis. The long molecule axes tend to be parallel to the phase symmetry axis just like in typical nematics. The nonzero order parameters are $\left\langle E_{00}^{(j)}\right\rangle=\left\langle P_{j}\left(n_{z}\right)\right\rangle$ for $j$ even. For the parallel field the phase has the symmetry $C_{\infty v}$ whereas for the perpendicular field the phase has the symmetry $C_{2 v}$. The expressions $U_{\mu \mu}^{j j}$ are nonzero

$$
\begin{aligned}
& \epsilon_{0} \chi_{x x}=\epsilon_{0} \chi_{y y}=\frac{N p^{2} \beta(2+S)}{V\left[6+\beta v_{1}(2+S)\right]}, \\
& \epsilon_{0} \chi_{z z}=\frac{N p^{2} \beta(1-S)}{V\left[3+\beta v_{1}(1-S)\right]} .
\end{aligned}
$$

Let us note that for $v_{1}=0$ the expressions $(6),(7)$ are recovered. For $v_{1}<0$ the susceptibility $\chi_{x x}$ diverges at the transition from the uniaxial nematic to the ferroelectric phase.

\subsection{The uniaxial ferroelectric phase}

For the zero field and for the parallel field the phase has the symmetry $C_{\infty v}$. The phase is oriented along the $x$ axis. The molecule dipoles (parallel to the short molecule axes) on average point to the same $x$ direction whereas the long molecule axes are distributed randomly in the $y-z$ plane. We have nonzero $S_{11}^{(1)}$ and $S_{20}^{(2)}=-\sqrt{3} S_{00}^{(2)}$. The nonzero order parameters are: $\left\langle E_{11}^{(1)}\right\rangle=\left\langle l_{x}\right\rangle,\left\langle E_{20}^{(2)}\right\rangle=-\sqrt{3}\left\langle E_{00}^{(2)}\right\rangle,\left\langle E_{02}^{(2)}\right\rangle,\left\langle E_{22}^{(2)}\right\rangle$. In the ideally ordered phase the values of the order parameters are: $\left\langle E_{11}^{(1)}\right\rangle=1,\left\langle E_{00}^{(2)}\right\rangle=1 / 4,\left\langle E_{02}^{(2)}\right\rangle=\left\langle E_{20}^{(2)}\right\rangle=-\sqrt{3} / 4$, $\left\langle E_{22}^{(2)}\right\rangle=3 / 4$. The most important nonzero elements $U_{\mu \nu}^{j k}$ are: $U_{11}^{11}, U_{00}^{11}, U_{-1,-1}^{11}, U_{12}^{12}, U_{10}^{12}, U_{01}^{12}, U_{-1,-2}^{12}, U_{22}^{22}, U_{20}^{22}$, $U_{00}^{22}, U_{11}^{22}, U_{-1,-1}^{22}, U_{-2,-2}^{22}$ :

$$
\begin{aligned}
& \epsilon_{0} \chi_{x x}=\frac{N p^{2} \beta}{V} \\
& \quad \times \frac{U_{11}^{11} A_{1}-U_{12}^{12} A_{2}+U_{10}^{12} A_{3}}{\left(1+\beta v_{1} U_{11}^{11}\right) A_{1}-\beta v_{1} U_{12}^{12} A_{2}+\beta v_{1} U_{10}^{12} A_{3}},
\end{aligned}
$$

where

$$
\begin{aligned}
A_{1} & =\left(1+\beta v_{2} U_{22}^{22}\right)\left(1+\beta v_{2} U_{00}^{22}\right) \\
& -\left(\beta v_{2} U_{02}^{22}\right)\left(\beta v_{2} U_{20}^{22}\right), \\
A_{2} & =\left(\beta v_{2} U_{21}^{21}\right)\left(1+\beta v_{2} U_{00}^{22}\right) \\
& -\left(\beta v_{2} U_{01}^{21}\right)\left(\beta v_{2} U_{20}^{22}\right) \\
A_{3} & =\left(\beta v_{2} U_{21}^{21}\right)\left(\beta v_{2} U_{02}^{22}\right) \\
& -\left(\beta v_{2} U_{01}^{21}\right)\left(1+\beta v_{2} U_{22}^{22}\right) .
\end{aligned}
$$

\subsection{The biaxial ferroelectric phase}

For the zero field and for the parallel field the phase has the symmetry $C_{2 v}$. The phase is oriented along the $x$ axis. The molecule dipoles on average point to the same $x$ direction whereas the long molecule axes are parallel to the $z$ direction. We have independent nonzero $S_{11}^{(1)}, S_{20}^{(2)}$, and $S_{00}^{(2)}$. The most important order parameters are: $\left\langle E_{11}^{(1)}\right\rangle,\left\langle E_{00}^{(2)}\right\rangle,\left\langle E_{02}^{(2)}\right\rangle,\left\langle E_{20}^{(2)}\right\rangle,\left\langle E_{22}^{(2)}\right\rangle$, and 
$\left\langle E_{-1,-1}^{(2)}\right\rangle$. In the ideally ordered phase the values of the order parameters are: $\left\langle E_{11}^{(1)}\right\rangle=1,\left\langle E_{00}^{(2)}\right\rangle=\left\langle E_{22}^{(2)}\right\rangle=1$, $\left\langle E_{02}^{(2)}\right\rangle=\left\langle E_{20}^{(2)}\right\rangle=0$. The susceptibility is described by Eq. (44).

\section{Results}

In this section we carry out calculations for different physical systems of polar molecules described by the considered model. The phase diagram of the model is shown in Fig. 1. Four phases are present: isotropic, uniaxial nematic, uniaxial ferroelectric, and biaxial ferroelectric. Dashed half-lines in the picture denote different physical systems with the fixed parameters $v_{1}$ and $v_{2}$. Four critical points from $C_{1}$ to $C_{4}$ are predicted. $C_{1}=(-3.0,-3.3)$ and $C_{2}=(-3.4,-3.8)$ are tricritical points where transition changes from second-order to

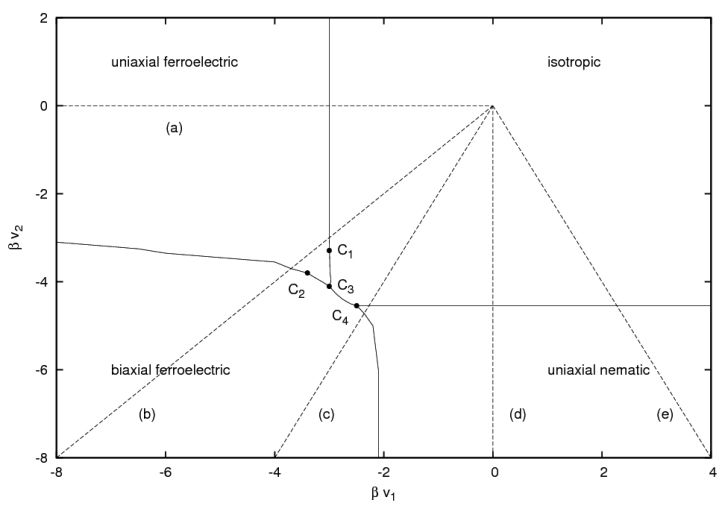

Fig. 1. Phase diagram of the model considered in the paper, where $v_{1}$ and $v_{2}$ are the parameters of molecular interactions, $1 / \beta=k_{\mathrm{B}} T$. Four phases are present: isotropic, uniaxial nematic, uniaxial ferroelectric, and biaxial ferroelectric. Four critical points from $C_{1}$ to $C_{4}$ are predicted. Dashed half-lines denote different physical systems: (a) $v_{2}=0$, (b) $v_{2}=v_{1}$, (c) $v_{2}=2 v_{1}$, (d) $v_{1}=0$, and (e) $v_{2}=-2 v_{1}$. For a given physical system on decreasing the temperature we are moving from the center $(0,0)$ to the edge of the figure.

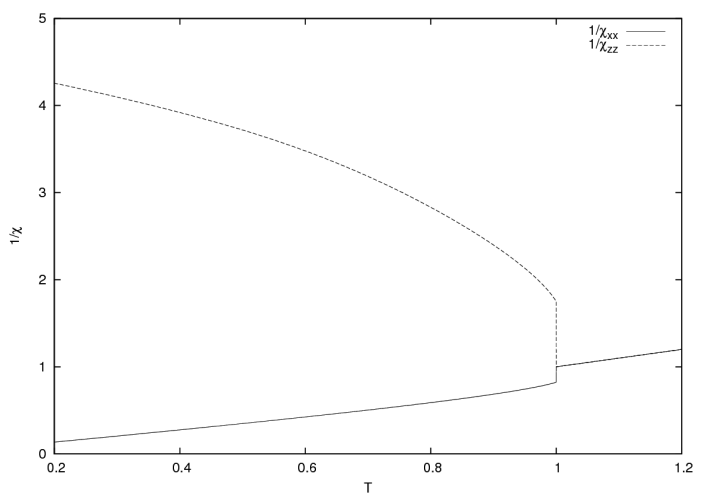

Fig. 2. Temperature dependence of the inverse susceptibility for $v_{1}=0$ [half line (d) in Fig. 1]. On decreasing the temperature the susceptibility splits into $\chi_{z z}<\chi_{x x}$. first-order. $C_{3}=(-3.0,-4.1)$ and $C_{4}=(-2.5,-4.54)$ are points where three phases coexist in equilibrium.

Let us start from the systems known from the previous studies [10]. For the case of $v_{2}=0$ [half line (a) in Fig. 1] we get the simple system with the second-order transition from the isotropic to the uniaxial ferroelectric phase at $T_{\mathrm{C}}=-v_{1} / 3 k_{\mathrm{B}}$. For the case of $v_{1}=0$ [half line (d) in Fig. 1] the interactions are uniaxial (small dipoles) and there is the first-order transition from the isotropic to the uniaxial nematic phase. The temperature dependence of the inverse susceptibility is presented in Fig. 2. In all pictures, $T$ denotes the dimensionless temperature. $T=1$ corresponds to the transition from the isotropic to the nematic or ferroelectric phase. The susceptibilities are expressed in $N p^{2} /\left(V \epsilon_{0}\left|v_{1}\right|\right)$. On decreasing the temperature the susceptibility splits into $\chi_{z z}<\chi_{x x} . \chi_{x x}$ runs to the infinity whereas $\chi_{z z}$ remains finite. This is typical for the uniaxial molecules with the dipole moment perpendicular to the symmetry axis.

In the system described by half line (b) in Fig. 1 there are the second-order transitions from the isotropic phase

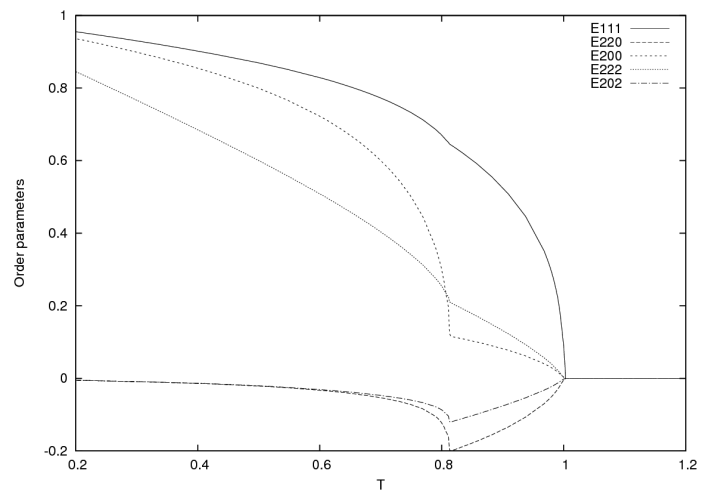

Fig. 3. Temperature dependence of the order parameters for $v_{1}=v_{2}$ [half line (b) in Fig. 1]. There are the second-order transitions from the isotropic phase to the uniaxial ferroelectric phase $(T=1)$ and next to the biaxial ferroelectric phase $(T=0.81)$.

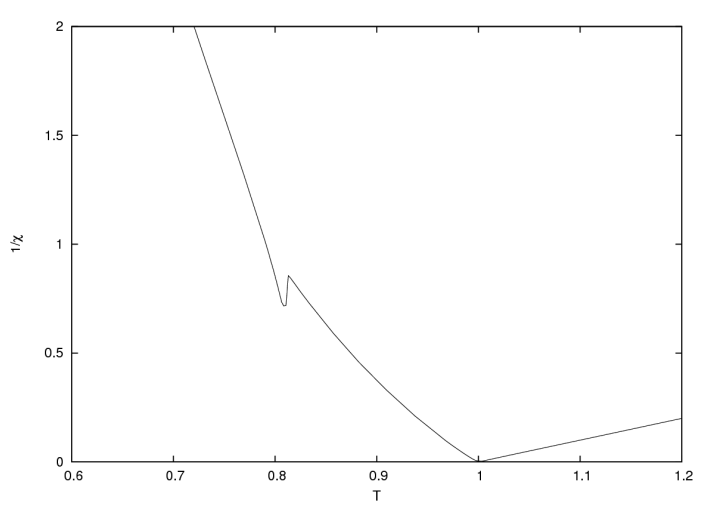

Fig. 4. Temperature dependence of the inverse susceptibility for $v_{1}=v_{2}$ [half line (b) in Fig. 1]. 


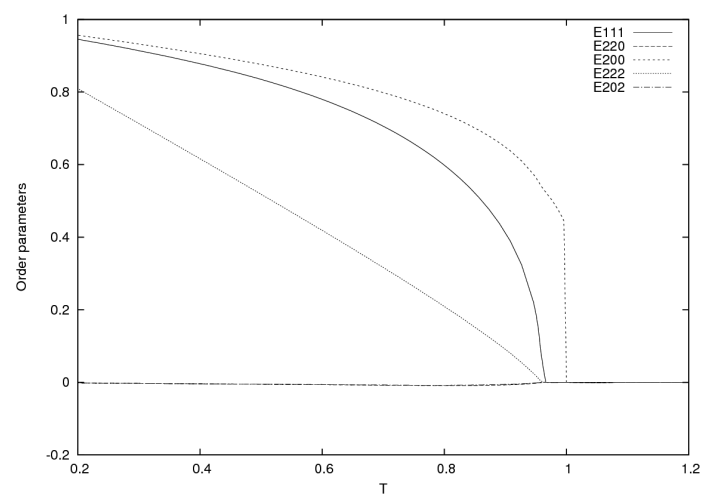

Fig. 5. Temperature dependence of the order parameters for $v_{2}=2 v_{1}$ [half line (c) in Fig. 1]. There is the first-order transition from the isotropic phase to the uniaxial nematic phase $(T=1)$ and the second-order transition from the uniaxial nematic phase to the biaxial ferroelectric phase $(T=0.96)$.

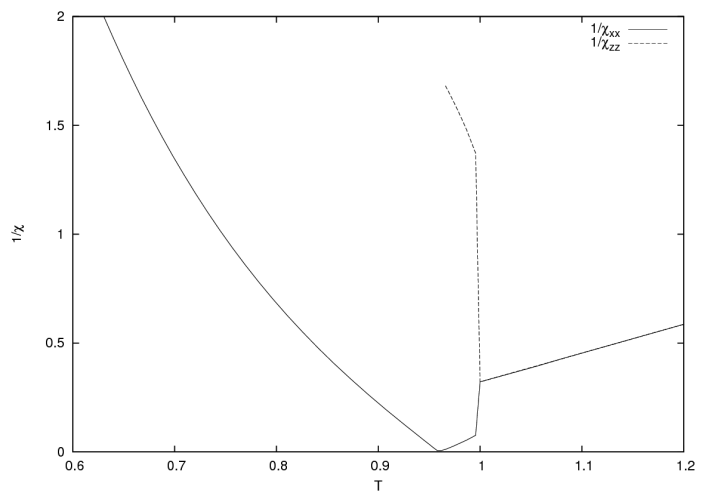

Fig. 6. Temperature dependence of the inverse susceptibility for $v_{2}=2 v_{1}$ [half line (c) in Fig. 1].

to the uniaxial ferroelectric phase $(T=1)$ and next to the biaxial ferroelectric phase $(T=0.81)$. The temperature dependence of the order parameters and the inverse susceptibility is presented in Figs. 3 and 4, respectively. We note that the susceptibility reveals the transition from the uniaxial ferroelectric to the biaxial ferroelectric phase.

A very interesting situation takes place in the system described by half line (c) in Fig. 1. The temperature dependence of the order parameters and the inverse susceptibility is presented in Figs. 5 and 6, respectively. On decreasing the temperature we meet the first-order transition from the isotropic to the uniaxial nematic phase $(T=1)$ and the second-order transition to the biaxial ferroelectric phase $(T=0.96)$. In the uniaxial nematic phase we have $\chi_{x x}>\chi_{y y}=\chi_{z z}$.

In the case of the system described by half line (e) in Fig. 1 two phases are present: isotropic and uniaxial nematic with the first-order transition between them. The order parameter $\left\langle E_{00}^{(2)}\right\rangle$ is the most important and the susceptibility is finite.

\section{Summary}

In this paper, we presented the statistical theory of the dielectric susceptibility of polar liquid crystals. The molecules were calamitic or bent-core with the permanent perpendicular dipole moment. We focused on spatially homogeneous (liquid) phases because the understanding and classifying of those phases is a prerequisite to study of more ordered phases, which in addition break translational symmetry.

TABLE

Liquid phases of banana-shaped molecules, their symmetries in the Schoenflies notation, and nonzero variables in the considered model. Four phases are present: isotropic $(\mathrm{I})$, uniaxial nematic $(\mathrm{N})$, uniaxial ferroelectric $(\mathrm{V})$, and biaxial ferroelectric $(\mathrm{V}+2)$.

\begin{tabular}{c|c|c}
\hline \hline Phase & Symmetry & Variables \\
\hline $\mathrm{I}$ & $O(3)$ & - \\
$\mathrm{N}$ & $D_{\infty h}$ & $S_{00}^{2}$ \\
$\mathrm{~V}$ & $C_{\infty v}$ & $S_{11}^{1}, S_{20}^{2}=-\sqrt{3} S_{00}^{2}$ \\
$\mathrm{~V}+2$ & $C_{2 v}$ & $S_{11}^{1}, S_{00}^{2}, S_{20}^{2}$
\end{tabular}

Liquid phases of bent-core molecules from the considered model are listed in Table I. Phase transition types obtained in the present paper can be summarized as follows:

1. $\mathrm{I} \rightarrow \mathrm{N}$ transition: first-order;

2. $\mathrm{I} \rightarrow \mathrm{V}$ transition: second-order or weakly first-order (second-order in [3]);

3. $\mathrm{I} \rightarrow \mathrm{V}+2$ transition: first-order (not present in [3]);

4. $\mathrm{N} \rightarrow \mathrm{V}+2$ transition: second-order;

5. $\mathrm{V} \rightarrow \mathrm{V}+2$ transition: second-order or weakly first-order (second-order in [3]).

Every transition has unique features which can be observed from the temperature dependence of the order parameters or susceptibilities. Generally, the classification by Lubensky and Radzihovsky [3] is completed. The results for the transitions $\mathrm{I} \rightarrow \mathrm{V}, \mathrm{I} \rightarrow \mathrm{V}+2$, and $\mathrm{V} \rightarrow \mathrm{V}+2$ was obtained also by Mettout et al. [11] in a theory with two vectors representing the six-dimensional order parameter associated with the transition from the isotropic liquid to a polar nematic phase.

Solid ferroelectrics and related materials are of great interest for applications in electronics and optoelectronics (materials for capacitors, piezotransducers, light modulators, light frequency converters, etc.). There is no fundamental reason that ferroelectric nematic phases should not exist. However, typical ferroelectric fluids are chiral smectic phases and related structures [12]. These layered phases lack the high fluidity and self healing characteristics that make nematics useful in electrooptical devices. 
Polar nematic phases of thermotropic liquid crystals are expected to exhibit much faster and easier response to an external electric field [13]. These materials may have interesting technological applications [14], and that is why a number of theoretical studies have focused on the possibility of realizing such phases. The effect of dipolar forces on the structure and thermodynamic of classical fluids was reviewed by Teixeira et al. [15]. Theoretical predictions and experimental observations concerning polar achiral mesomorphic phases were presented by Blinov [16].

The model fluids consisting of cylindrically symmetric molecules with an electric dipole moment parallel to the molecule symmetry axes were studied in many papers [10, 17-19]. It was shown that the uniaxial ferroelectric phase can be formed by disk-like molecules which have reasonable electric dipole moments. However, steric interactions between asymmetric molecules generally disfavor ferroelectric nematics. Sometimes more ordered columnar or crystal phases are expected. The mean-field theory was used to analyze the second-harmonic generation in a lyotropic liquid crystalline system [19] and the presence of the ferroelectric ordering was revealed. In computer simulations of a system of tapered molecules the following sequence of phases was obtained on cooling the sample: isotropic, apolar uniaxial nematic, polar uniaxial nematic and smectic phases [20]. The polar nematic phase was also stable after introduction of a small axial dipole, thus yielding a ferroelectric nematic. The same phases were found in the off-lattice Krieger-James model of ordered fluids [21]. Dipolar hard spherocylinders were studied by means of the Monte Carlo simulation [22] and numerical analyses [23], where the polar nematic phase was obtained when molecule dipole moments reached a critical value.

Solid ferroelectrics usually have domain structure because a uniformly polarized state is unstable. It was shown that the polar nematic phase is also unstable and a helical structure is acquired in order to reduce the electrostatic energy [24]. The helical pitch depends on the geometric size of the sample, the absolute value of the spontaneous polarization, and the elastic moduli.

Polar biaxial liquid crystalline phases were discussed by Brand et al. [25]. The polar biaxial nematic phase appeared to be a rather natural candidate for biaxial nematic phases made of banana-shaped molecules. The possibility of an occurrence of spontaneous bend and splay was concluded. In 2008 Vanakaras and Photinos [26] suggested the possibility of polar ordering within the biaxial clusters in the macroscopically uniaxial nematic phase composed of bent-core molecules. Under the action of an electric field, the biaxial ferroelectric phase could be obtained from the collective alignment of the domains. The existence of biaxial and uniaxial ferroelectric phases requires further experimental confirmation.

An extended version of the considered model can be obtained from the Straley model of biaxial nematics [27] by the inclusion of our $P_{1}\left(\boldsymbol{l}_{1} \cdot \boldsymbol{l}_{2}\right)$ term or the $P_{1}\left(\boldsymbol{n}_{1} \cdot \boldsymbol{n}_{2}\right)$ term. Then the biaxial nematic $N+2$ phase with $D_{2 h}$ symmetry would be present and new transition types would be possible: $\mathrm{I} \rightarrow \mathrm{N}+2, \mathrm{~N} \rightarrow \mathrm{N}+2$, or $\mathrm{N}+2 \rightarrow \mathrm{V}+2$.

\section{Appendix A}

Below we list the properties of the functions $E_{\mu \nu}^{(j)}$. The functions can be used to describe any physical quantity which depends on the three Euler angles.

1. The definition is

$$
\begin{aligned}
& E_{\mu \nu}^{(j)}(R)=\left(\frac{1}{\sqrt{2}}\right)^{2+\delta_{0 \mu}+\delta_{0 \nu}} \frac{1}{2}[(1+\mathrm{i})+(1-\mathrm{i}) \\
& \quad \times \operatorname{sgn}(\mu) \operatorname{sgn}(\nu)]\left[D_{\mu \nu}^{(j)}(R)+\operatorname{sgn}(\mu) \operatorname{sgn}(\nu)\right. \\
& \quad \times(-1)^{\mu+\nu} D_{-\mu,-\nu}^{(j)}(R)+\operatorname{sgn}(\nu)(-1)^{\nu} \\
& \left.\quad \times D_{\mu,-\nu}^{(j)}(R)+\operatorname{sgn}(\mu)(-1)^{\mu} D_{-\mu, \nu}^{(j)}(R)\right],
\end{aligned}
$$

where $R=(\phi, \theta, \psi)$ (the three Euler angles), $j$ is a non-negative integer, $\mu$ and $\nu$ are integers. Functions $D_{\mu \nu}^{(j)}$ are standard rotation matrix elements [28] and

$$
\operatorname{sgn}(x)=\left\{\begin{array}{r}
1 \text { for } x \geq 0 \\
-1 \text { for } x<0
\end{array}\right.
$$

Let us note that

$$
\operatorname{sgn}(-x)=-\operatorname{sgn}(x)+2 \delta_{0 x} .
$$

2. The functions $E_{\mu \nu}^{(j)}$ are real.

3. The functions satisfy the orthogonality relations

$$
\int \mathrm{d} R E_{\mu \nu}^{(j)}(R) E_{\rho \sigma}^{(k)}(R)=\delta_{j k} \delta_{\mu \rho} \delta_{\nu \sigma} 8 \pi^{2} /(2 j+1) .
$$

4. Let us assume that the three Euler angles $R=$ $(\phi, \theta, \psi)$ determine the orientation of the three unit orthogonal vectors $(\boldsymbol{l}, \boldsymbol{m}, \boldsymbol{n})$. The functions $E_{\mu \nu}^{(j)}$ can be expressed in terms of the vector coordinates

$$
\begin{aligned}
& E_{00}^{(1)}(R)=n_{z}, \\
& E_{01}^{(1)}(R)=l_{z}, \\
& E_{10}^{(1)}(R)=n_{x}, \\
& E_{11}^{(1)}(R)=l_{x} \\
& E_{00}^{(2)}(R)=\frac{1}{2}\left(-1+3 n_{z}^{2}\right), \\
& E_{02}^{(2)}(R)=\frac{\sqrt{3}}{2}\left(-1+n_{z}^{2}+2 l_{z}^{2}\right), \\
& E_{20}^{(2)}(R)=\frac{\sqrt{3}}{2}\left(-1+n_{z}^{2}+2 n_{x}^{2}\right), \\
& E_{22}^{(2)}(R)=\frac{1}{2}\left(-3+n_{z}^{2}+2 l_{z}^{2}+2 n_{x}^{2}+4 l_{x}^{2}\right) .
\end{aligned}
$$




\section{References}

[1] P.G. de Gennes, J. Prost, The Physics of Liquid Crystals, Clarendon Press, Oxford 1993.

[2] H. Takezoe, Y. Takanishi, Jpn. J. Appl. Phys. 45, 597 (2006).

[3] T.C. Lubensky, L. Radzihovsky, Phys. Rev. E 66, 031704 (2002).

[4] P.J. Camp, M.P. Allen, A.J. Masters, J. Chem. Phys. 111, 9871 (1999).

[5] Y. Lansac, P.K. Maiti, N.A. Clark, M.A. Glaser, Phys. Rev. E 67, 011703 (2003).

[6] M.A. Bates, G.R. Luckhurst, Phys. Rev. E 72, 051702 (2005).

[7] M.A. Bates, Chem. Phys. Lett. 437, 189 (2007).

[8] W. Maier, G.Z. Meier, Z. Naturforsch. A 16, 262, 470, 1200 (1961).

[9] A. Kapanowski, T. Wietecha, Phys. Rev. E 71, 021710 (2005).

[10] A. Kapanowski, T. Wietecha, Acta Phys. Pol. A 106, 51 (2004).

[11] B. Mettout, P. Toledano, H. Takezoe, J. Watanabe, Phys. Rev. E 66, 031701 (2002).

[12] C. Zannoni, J. Mater. Chem. 11, 2637 (2001).

[13] O. Francescangeli, V. Stanic, S.I. Torgova, A. Strigazzi, N. Scaramuzza, C. Ferrero, I.P. Dolbnya, T.M. Weiss, R. Berardi, L. Muccioli, S. Orlandi, C. Zannoni, Adv. Funct. Mater. 19, 2592 (2009).

[14] D. Guillon, Adv. Chem. Phys. 113, 1 (2000).
[15] P.I.C. Texeira, J.M. Tavares, M.M. Telo da Gama, J. Phys., Condens. Matter 12, R411 (2000).

[16] L.M. Blinov, Liq. Cryst. 24, 143 (1998).

[17] P. Palffy-Muhoray, M.A. Lee, R.G. Petschek, Phys. Rev. Lett. 60, 2303 (1988).

[18] M. Baus, J.-L. Colot, Phys. Rev. A 40, 5444 (1989).

[19] B. Park, J.W. Wu, H. Takezoe, Phys. Rev. E 63, 021707 (2001).

[20] R. Berardi, M. Ricci, C. Zannoni, Chem. Phys. Chem. 2, 443 (2001)

[21] P.I.C. Teixeira, Liq. Cryst. 25, 721 (1998).

[22] D. Levesque, J.J. Weis, G.J. Zarragoicoechea, Phys. Rev. E 47, 496 (1993).

[23] J. Lee, S.D. Lee, Mol. Cryst. Liq. Cryst. 254, 395 (1994).

[24] A.G. Khachaturyan, J. Phys. Chem. Solids 36, 1055 (1975).

[25] H.R. Brand, P.E. Cladis, H. Pleiner, Int. J. Eng. Sci. 38, 1099 (2000).

[26] A.G. Vanakaras, D.J. Photinos, J. Chem. Phys. 128, 154512 (2008).

[27] J.P. Straley, Phys. Rev. A 10, 1881 (1974).

[28] A.R. Edmonds, Angular Momentum in Quantum Mechanics, Princeton U.P., Princeton, NJ 1957. 